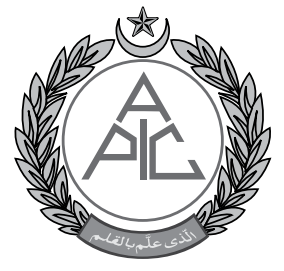

${ }^{1}$ Consultant Anesthetist and Intensive Care, Shaukat Khanum Memorial Cancer Hospital and Research Centre (SKMCH\&RC), Lahore (Pakistan)

Correspondence:

Dr Saad Ur Rehman, Shaukat

Khanum Memorial Cancer Hospital and Research Centre, Johar Town, Lahore (Pakistan); Phone No. Work: +924235905000, ext 5116; Cell: +923434545963; Email: saad rehman@yahoo.com

Received: 8 Sep 2018

Reviewed: 16 Sep 2018

Accepted: 15 Oct 2018

\section{Febrile neutropenia in intensive care: a challenge for the modern day intensivist}

\author{
Saad Ur Rehman ${ }^{1}$, Ahsun Waqar Khan ${ }^{1}$
}

\begin{abstract}
Febrile Neutropenia (FN) is a fairly common complication of cancer chemotherapy. It leads to delays in cancer treatment and worsens prognosis. Patients with $\mathrm{FN}$ are frequently admitted to intensive care with organ specific complications of FN. The optimal management of critically ill patients with FN necessitates expertise in oncology, critical care, and infectious diseases. Intensive care specialists therefore have to be familiar with key principles of care for critically ill patients with cancer and FN. This review provides an overview of the pathophysiology, definition, management and prognostic factors of critically ill patients with FN.
\end{abstract}

Key words: Febrile neutropenia; Critical care; Sepsis

Citation: Rehman SU, Khan AW. Febrile neutropenia in intensive care: a challenge for the modern day intensivist. Anaesth Pain \& Intensive Care 2018;22 Suppl 1:S102-S105

\section{INTRODUCTION}

The management of febrile neutropenia (FN) and neutropenic sepsis is difficult and frustrating, so much so that just about two decades ago, intensivists were debating whether these patients should even be admitted to the critical care unit. ${ }^{1}$ According to current estimates the hospital survival of patients with neutropenic sepsis admitted to intensive care has improved to $50 \% .^{2}$ As the survival of patients with cancer increases, more and more patients with malignancies will be admitted to critical care, hence an understanding of neutropenic sepsis and its management is paramount for intensivists.

\section{DEFINITION}

There are several definitions of FN. According to the European Society for Medical Oncology (ESMO), FN is defined as: 'an oral temperature of $>38.5^{\circ} \mathrm{C}$ or two consecutive readings of $>38.0^{\circ} \mathrm{C}$ for $2 \mathrm{~h}$ and an absolute neutrophil count (ANC) of $<0.5 \times 10^{9} \mathrm{~L}$ or expected to fall $<0.5 \times 10^{9} \mathrm{~L}^{3}$ According to the Infectious Diseases Society of America, FN is defined as "single oral temperature of $\geq 38.0^{\circ} \mathrm{C}\left(101.4^{\circ} \mathrm{F}\right)$ for $\geq$
$1 \mathrm{~h}$ ". Neutropenia is defined as "a neutrophil count of $<500$ cells per cubic mm or a count of less than 1000 cell per cubic $\mathrm{mm}$ with a predicted decrease of less than 500 cells per cubic mm".

The incidence of $\mathrm{FN}$ varies between 10 and $50 \%$ in solid tumors and is reportedly $\geq 80 \%$ in hematological malignancies. ${ }^{4} \mathrm{FN}$ warrants immediate hospitalization and management. It leads to delays, reduction and change of chemotherapeutic agents which adversely affect outcomes and disease free period. ${ }^{5,6}$

\section{PATHOPHYSIOLOGY}

Neutropenia complicates almost $24 \%$ of patients with solid organ tumors and hematological malignancies. It is associated with both chemotherapy and radiotherapy. Chemotherapeutic drugs kill and suppress all the cells that have a high rate of division. Thus they affect blood cells, bone cells, and neutrophil cells. Actinomycin, asparaginase, cytarabine, busulfan, cisplatin, daunorubicin, etoposide, fluorouracil, ifosfamide, and methotrexate are highly associated with neutropenia development. 
For chemotherapy to be effective it needs to be administered on continuous basis. However, chemotherapy toxicity results in the destruction of all cell lines and is stopped for sufficient intervals so as to allow normal cells to recover. The duration of chemotherapy administration is known as chemotherapy cycle. Neutropenia incidence is mainly and highly associated with the first cycle of the chemotherapy more than the other or subsequent cycles.

The majority of causative organisms involved are bacterial; prolonged neutropenia, hospital stay and antibiotic use predispose to resistant organisms. Organisms include gram-positive bacteria, such as coagulase-negative staphylococci, Staphylococcus aureus, Enterococcus species, and Streptococcus species. More recently, drug-resistant gram-negative organisms including pseudomonas aeruginosa, acinetobacter species, stenotrophomonas maltophilia, escherichia coli, and klebsiella species have been identified as etiologic agents. In cases of prolonged neutropenia and persistent infection, fungi like Candida species, Aspergillus species or viral infections can be seen. ${ }^{7}$

\section{PRESENTATION}

Presentation is usually to the emergency department with high grade fevers however atypical presentations with patient feeling generally unwell are also fairly common. ${ }^{8}$ Clinical deterioration may be seen in patients admitted to wards as well after administration of chemotherapy. Some of these patients are on corticosteroids and clinical signs of overt bacteremia may be masked.

\section{MANAGEMENT}

The benefits of a detailed history and extensive physical examination cannot be over emphasized. Recent infection, use of previous antibiotics for prophylaxis, treatment and previous culture sensitivities should be reviewed. An extensive review of systems should be done. Any hemodynamic compromise or organ failure should prompt activation of sepsis pathway and intensive care involvement. Tonsillar hypertrophy and site of indwelling catheters should be checked. Investigations should include biomarkers of infection including C-reactive protein, at least 2 sets of blood cultures (both peripheral and indwelling catheters) urine, sputum, feces. Swabs from any skin wounds and broken mucosal membranes should be taken and chest $\mathrm{x}$-ray ordered. ${ }^{9}$

\section{Risk Stratification:}

All patients with FN should be stratified for high risk of serious complications. Scoring systems evaluate for development of serious complications, duration and choice of antibiotics and in hospital mortality. Patients at high risk may develop organ failure necessitating intensive care admission. Scoring systems such as The Multinational Association for Supportive Care in Cancer (MASCC) risk index score and The Clinical Index of Stable Febrile Neutropenia (CISNE) score are used commonly for risk stratification in neutropenic patients. ${ }^{10,11}$

\section{Antibiotics:}

In high risk patients, the initial choice of antibiotics should be broad spectrum beta lactam antibiotics like piperacillin/tazobatam, cefipime or carbapenems. Gram-positive cover is usually not required but should be considered in patients with indwelling catheters, skin/soft tissue infections and patients going into multiorgan failure.

In patients with pneumonia, atypical organisms like mycoplasma and Legionella should be covered with macrolides. Pneumocystis jerovecii and mycobacteria should be considered in all patients with atypical infiltrates and rapid progression to respiratory failure. These patients should undergo a bronchoalveolar lavage and started on treatment dose of co-trimoxazole.

Antifungals should be considered when fever does not respond to broad spectrum antibiotics. Liposomal amphotericin B or an echinocandin antifungal such as caspofungin are appropriate first-line treatment if the patient has already been exposed to an azole or if the patient is known to be colonized with non albicans candida. Fluconazole is not usually used but can be given first line provided the patient is at low risk of invasive aspergillosis. Once started, antifungal treatment should be continued until neutropenia has resolved, or for at least 14 days in patients with a demonstrated fungal infection e.g. lung infiltrates.

\section{Intensive Care Management:}

Patients with FN are usually admitted to intensive care for sepsis, respiratory failure and complications of neutropenic enterocolitis. Data is scarce on prognostic risk factors; however old age, multi organ involvement and patients with bone marrow transplant and hematopoietic stem cell transplant have a poorer prognosis. Severity of neutropenia usually does not affect outcomes.

Patients should be admitted to critical care early rather than waiting for multiorgan failure to develop. Delayed ICU admission is associated with worse outcomes. $^{12}$

The management of these patients should be multi- 
disciplinary with intensivist taking the lead working in consultation with oncologist and microbiologist/ infectious disease specialist. Early involvement of palliative care team should be sought in cases with multi organ involvement. Sepsis and Septic shock should be managed according to severe sepsis guidelines. Fever trends and markers of infection should be monitored closely and any sign of clinical deterioration should prompt an escalation of antibiotic cover. Source control should take place early; in children sinus infections should not be ignored.

In cases of respiratory failure, even with rough estimates mortality is more than $50 \%$ population. Lung protective ventilation should be employed. Diffuse lung infiltrates have a worse prognosis than lobar infiltrates. Traditionally intensivists favored noninvasive ventilation (NIV) for cancer patients however NIV failure is associated with worse prognosis and early intubation is favored over NIV except in patients with COPD and pulmonary edema. In single organ failure it may be a good practice to give a short trial of NIV and assess for improvement. ${ }^{13,14}$ Bronchoalveolar lavage adds value both in diagnosis and management of patients with respiratory failure. Weaning can be difficult and usual length of stay is about 3 weeks. Persistent neutropenia and multiorgan failure adversely affect outcomes. ${ }^{13}$

Prophylactic Granulocyte-Colony stimulating factor (G-CSF) is used for prevention of neutropenia in high risk cases. It is not recommended in routine management of patients with established FN but is considered in high risk patients. ${ }^{15}$

Gut failure in the form of diarrhea, distention, abdominal pain, cramping and bloody stools can be signs of neutropenic colitis. This is caused by neutropenia and breakage of mucosal layer of gut. Radiological imaging in form of CT confirms the diagnosis although USG can be useful as well. Management is supportive with bowel rest, parenteral nutrition, correction of coagulopathy and antibiotics. Patients should be monitored for signs of peritonitis, perforation or worsening of symptoms which require surgical intervention. ${ }^{16}$

Finally every cancer patient will not benefit from intensive care. The decision to initiate multi organ support should be individualized and discussed with oncologist and patient as well. Treatment goals of patients should be discussed regularly and the oncologist involved along with the patient in decision making regarding continuation and cessation of life supporting treatment.

\section{CONCLUSION}

The management of a patient with $\mathrm{FN}$ in critical care is a collaborative effort, and close cooperation between critical care, oncology and infectious diseases team is essential. The principles of management include early diagnosis and start of empirical antibiotics, risk stratification and monitoring response to management, antibiotics and assessment of organ dysfunction. Early involvement of critical care team and timely interventions can improve prognosis of these patients.

Conflict of interest: Nil

Authors' contribution:

SUR: Concept, Review of Literature, Manuscript writing

AWK: Manuscript review and Editing

\section{REFERENCES}

1. Benoit DD, Vandewoude KH, Decruyenaere JM, Hoste EA, Colardyn FA. Outcome and early prognostic indicators in patients with a hematologic malignancy admitted to the intensive care unit for a life-threatening complication. Crit Care Med. 2003 Jan 1;31(1):104-12. [PubMed]

2. Azoulay E, Mokart D, Pène F, Lambert $J$, Kouatchet A, Mayaux J, Vincent $F$, Nyunga $M$, Bruneel $F$, Laisne LM, Rabbat A. Outcomes of critically ill patients with hematologic malignan- cies: prospective multicenter data from France and Belgium-a groupe de recherche respiratoire en reanimation onco-hematologique study. Journal of Clinical Oncology. 2013 Jun 10;31(22):2810-8. [PubMed]

3. De Naurois J, Novitzky-Basso I, Gill MJ, Marti FM, Cullen MH, Roila F, ESMO Guidelines Working Group. Management of febrile neutropenia: ESMO clinical practice guidelines. Annals of Oncology. 2010 May 1;21(suppl_5):v252-6. [PubMed]
4. Klastersky J. Management of fever in neutropenic patients with different risks of complications. Clinical Infectious Diseases. 2004 Jul $15 ; 39$ (Supplement_1):S32-7. [PubMed]

5. Wolff D, Culakova E, Poniewierski MS, Lyman GH, Dale DC, Crawford J. Predictors of chemotherapy-induced neutropenia and its complications: results from a prospective nationwide registry. The journal of supportive oncology. 2005;3(6 Suppl 4):24-5. 


\section{febrile neutropenia in intensive care}

\section{[PubMed]}

6. Crawford J, Wolff DA, Culakova E, Poniewierski MS, Selby C, Dale DC, Lyman GH. First cycle risk of severe and febrile neutropenia in cancer patients receiving systemic chemotherapy: results from a prospective nationwide study. Blood. 2004 Dec 4;104(11):607a. [Free full text]

7. Cardoso LT, Grion CM, Matsuo T, Anami EH, Kauss IA, Seko L, et all. Impact of delayed admission to intensive care units on mortality of critically ill patients: a cohort study. Critical care. 2011 Feb;15(1):R28. [PubMed]

8. Lucas AJ, Olin JL, Coleman MD. Management and Preventive Measures for Febrile Neutropenia. Pharmacy and Therapeutics. 2018 Apr;43(4):228. [PubMed]

9. Klastersky J, Paesmans M. The Multinational Association for Supportive Care in Cancer (MASCC) risk index score: 10 years of use for identifying low-risk febrile neutropenic cancer patients. Supportive care in cancer. 2013 May 1;21(5):1487-95. [PubMed]

10. Coyne CJ, Le V, Brennan JJ, Castillo EM, Shatsky RA, Ferran K, Brodine S, Vilke GM. Application of the MASCC and CISNE risk-stratification scores to identify low-risk febrile neutropenic patients in the emergency department. Annals of emergency medicine. 2017 Jun 1;69(6):755-64. [PubMed]

11. Freifeld AG, Bow EJ, Sepkowitz KA, Boeckh MJ, Ito Jl, Mullen CA, et all. Clinical practice guideline for the use of antimicrobial agents in neutropenic patients with cancer: 2010 update by the Infectious Diseases Society of America. Clinical infectious diseases. 2011 Feb 15;52(4):e56-93. [PubMed]

12. Cardoso LT, Grion CM, Matsuo T, Anami EH, Kauss IA, Seko L, et all. Impact of delayed admission to intensive care units on mortality of critically ill patients: a cohort study. Critical care. 2011 Feb;15(1):R28. [PubMed]
13. Mokart D, van Craenenbroeck T, Lambert J, Textoris J, Brun JP, Sannini $A$, et al. Prognosis of acute respiratory distress syndrome (ARDS) in neutropenic cancer patients. European Respiratory Journal. 2012 Jan 1:erj01506-2011. [PubMed]

14. Schnell D, Lemiale V, Azoulay É. Noninvasive mechanical ventilation in hematology patients: let's agree on several things first. Critical care. 2012 Dec;16(6):175. [PubMed]

15. Smith TJ, Bohlke K, Lyman GH, Carson KR, Crawford J, Cross SJ, et all. Recommendations for the use of WBC growth factors: American Society of Clinical Oncology clinical practice guideline update. Journal of Clinical Oncology. 2015 Jul 13;33(28):3199212. [PubMed]

16. Nesher L, Rolston KV. Neutropenic enterocolitis, a growing concern in the era of widespread use of aggressive chemotherapy. Clinical infectious diseases. 2012 Nov 29;56(5):711-7. [PubMed] 